\title{
Ciclos largos de nivel de vida biológico en España (1750-1950): propuesta metodológica y evidencias locales
}

\section{Long-term cycles of biological living standards in Spain (1750-1950): a methodological proposal and local evidence}

\author{
ANTONIO D. CÁMARA Y JOAN GARCÍA ROMÁN \\ Universitat Autónoma de Barcelona y Centre d'Estudis Demogràfics
}

\begin{abstract}
RESUMEN
Este trabajo presenta una aplicación de la regresión logística para analizar datos antropométricos procedentes del reclutamiento militar desde el Antiguo Régimen. El objetivo es obtener información sobre el signo de las tendencias de estatura a largo plazo. Los datos proceden de dos localidades del sur de España y pertenecen a generaciones masculinas nacidas desde mediados del siglo XVIII hasta mediados del siglo Xx. Esos datos se han analizado en agrupaciones amplias de cohortes controlando el mínimo de estatura exigido, la edad y el municipio de residencia. Los resultados muestran un ciclo de deterioro del estado nutricional de las cohortes nacidas a lo largo

del siglo XIX con respecto a las nacidas antes de 1800. El descenso de la estatura es estadísticamente significativo para la agrupación de cohortes 18501899 mientras que las cohortes nacidas a partir de 1900 muestran una clara recuperación. Puesto que las series de estatura locales elaboradas hasta el momento en España desde mediados del siglo XIX muestran tendencias y valores razonablemente homogéneos, creemos que

estos resultados pueden constituir una primera

\section{ABSTRACT}

This work proposes an applied methodology based on the logistic regression to establish the sign of long-term trends in height from male anthropometric data collected since the Ancient Regime when mandatory conscription system was implemented in Spain. Data refer to male cohorts born from mid $18^{\text {th }}$ century to mid $20^{\text {th }}$ century. Broad cohort aggregations and control on the minimum required height as well as on age and municipality serve to implement the logistic regression model.

Results display a downward trend in stature for cohorts born throughout the $19^{\text {th }}$ century taking those born prior to 1800 as the reference. These results are statistically significant for the cohort group 1850-1899 as well as for those born after 1900 who display a clear recovery. Given that the $19^{\text {th }}$-century local series constructed in Spain up to now show similar trends, we think that these outcomes may serve as a first valid approach to the trends followed by the nutritional status prior to the socioeconomic modernization process experienced by Spain.
\end{abstract} aproximación válida a las tendencias seguidas por el estado nutricional en la fase previa a la modernización socioeconómica del país.

PALABRAS CLAVE: Niveles de vida biológicos, Antropometría, España, siglos XVIII-XX

Código JEL: I39, I19, N50, P25

KEY WORDS: Biological living standards, Anthropometrics, Spain, 18-20th centuries

JEL Codes: I39, I19, N50, P25 


\section{Introducción y objetivos ${ }^{1}$}

$\mathrm{L}$

os indicadores biosanitarios han constituido en las últimas décadas una fórmula bastante atractiva para medir el impacto de los procesos de cambio socioeconómico sobre la población. Con su utilización la Historia Económica ha logrado aproximaciones más poliédricas a la evolución del nivel de vida durante esos procesos constatándose, por ejemplo, la imperfecta relación entre el crecimiento económico y determinados componentes biológicos del bienestar humano como la salud. De este modo los estudios sobre mortalidad, esperanza de vida, o estado nutricional, elementos incluidos en el concepto más amplio de nivel de vida biológico ${ }^{2}$, son ya un clásico en la disciplina. En concreto, el éxito de los trabajos de Antropometría Histórica en el campo de la Historia Económica ha consistido en el potencial que ofrece la estatura adulta como indicador sintético de las condiciones ambientales en las que se desarrolla la vida durante la infancia y la adolescencia hasta que se completa el proceso de crecimiento físico ${ }^{3}$. Así, la estatura generacional (representada por la media de la de los individuos que tienen como acontecimiento común su año de nacimiento) interesa por ser un indicador de resultado del llamado estado nutricional neto. Éste, a modo de contabilidad, contempla las entradas y salidas energéticas del organismo (representadas respectivamente por la ingesta de alimentos y el gasto energético derivado principalmente del metabolismo basal, el esfuerzo físico y las enfermedades padecidas).

España es uno de los países con mayor volumen de datos antropométricos disponibles procedentes fundamentalmente del reclutamiento militar por quintas implantado durante el último tercio del siglo XVIII. Esto explica en parte la trayectoria tan fructífera seguida por la historia antropométrica española durante los últimos años y su importante contribución al complejo debate sobre los niveles de vida durante las primeras etapas del crecimiento económico español a lo largo del siglo XIX ${ }^{4}$. Los datos locales y nacionales de estatura estudiados hasta el momento (algunos conformando series temporales continuas) muestran un tránsito angosto hacia la consecución de un balance nutricional positivo y estable desde finales del siglo XIX y durante las primeras décadas del siglo $\mathrm{XX}^{5}$. Además, han revelado ciclos de estancamiento e incluso descenso de la estatura coincidiendo con periodos de crecimiento económico, integración de los mercados y desarrollo del capitalismo agrario en sintonía con lo ocurrido en otros países occidentales durante sus respectivos procesos de modernización ${ }^{6}$. Las evidencias en este sentido no resultaban demasiado novedosas para el ámbito urbano puesto que otros indicadores como la mortalidad ya habían puesto de manifiesto las consecuencias negativas que la urbanización acelerada durante el siglo XIX había tenido para la salud de la población en determinadas regiones europeas. Lo relativamente sorprendente fue comprobar que estos indicadores alternativos de nivel de vida

[Fecha de recepción del original, 26 de marzo de 2009. Versión definitiva, 1 de diciembre de 2009].

1 El presente trabajo se ha realizado gracias a recursos provistos por el proyecto I+D Crecimiento, nutrición y bienestar en España. La influencia de los procesos socioeconómicos a largo plazo en los niveles de vida biológicos y la salud (ref. SEJ2007-67613; financiado por el Ministerio de Educación y Ciencia) así como por el Centre d’Estudis Demogràfics de Barcelona.

Komlos (1994).

Tanner (1986); Bogin (1988).

Martínez Carrión (2001).

Martínez Carrión y Pérez Castejón (2002); Quiroga (2002); Cámara (2007); Puche Gil (2005); García Montero (2009); Ramón i Muñoz (2009).

Floud, Watcher y Gregory (1990); Cuff (2005). 
también experimentaron fases de deterioro entre la población rural durante la transición a la sociedad moderna ${ }^{7}$. Es más, en el caso español que no asistió a un proceso tan intenso de industrialización y urbanización, estudios recientes han cuestionado la hipótesis clásica de la penalización urbana durante el proceso de modernización y crecimiento económico del siglo $\mathrm{XIX}^{8}$.

El interés mostrado por la Historia Antropométrica española en la elaboración y análisis de tendencias durante la segunda mitad del siglo XIX es comprensible ya que es el periodo sobre el que se ha centrado el debate de la Historia Económica y la Historia Agraria acerca del atraso español en el contexto europeo. Son décadas clave en el desarrollo socioeconómico del país y también es el periodo en el que se diversifican las fuentes para estudiar las macromagnitudes del crecimiento económico coincidiendo con la consolidación del Estado moderno en España. No obstante, los datos antropométricos disponibles desde mediados del Ochocientos no permiten realizar demasiadas valoraciones acerca de las potenciales implicaciones del proceso de cambio socioeconómico sobre el estado nutricional si no son referenciados respecto a periodos previos. Dicho de otro modo, ignoramos por el momento si las bajas estaturas de las generaciones masculinas españolas nacidas a mediados del siglo XIX responden a una mejora, por leve que fuera, a un periodo de estancamiento o a un ciclo de deterioro del nivel de vida biológico con respecto al Antiguo Régimen y la transición a la sociedad moderna. Es cierto que se ha constatado un ciclo de descenso de la estatura generacional entre 1840 y 1870 aproximadamente pero la fecha de partida es demasiado avanzada como para pasar a analizar las implicaciones del proceso de transición del Antiguo Régimen a la sociedad moderna sobre el nivel de vida biológico de la población. Creemos, por tanto, que uno de los intereses principales de la Historia Antropométrica española en estos momentos es obtener datos y tendencias previas para interpretar adecuadamente los resultados obtenidos a partir de 1840 aproximadamente.

La tarea de prolongar las series antropométricas hacia el pasado no resulta fácil por el menor desarrollo del aparato estadístico estatal y la naturaleza mucho más compleja de las fuentes documentales que contienen los datos antropométricos ${ }^{9}$. Lo que se ha podido constatar hasta el momento, a nivel local, son ciclos cortos de mejora y deterioro de la estatura durante la segunda mitad del siglo XVIII ${ }^{10}$ sin que de momento se haya desarrollado una metodología general para reconstruir e interpretar las tendencias de estatura en ámbitos más amplios. Este trabajo pretende contribuir en este sentido presentando una metodología aplicada de análisis de datos antropométricos que permite utilizar la información disponible para las generaciones nacidas en las postrimerías del Antiguo Régimen (información no integrable en series temporales de estatura por la naturaleza de las fuentes tal y como se explicará más adelante).

Primero se procede a una contextualización del nivel de vida biológico en España en la segunda mitad del siglo XIX con referencias útiles que ayudarán a entender el valor potencial que tienen los ensayos sobre periodos anteriores. Después se presenta información general sobre las comunidades de las que proceden los datos antropométricos utilizados en el análisis. Por último se presentan y comentan los resultados obtenidos y se plantean futuras líneas de investigación que pueden apoyarse en esta metodología aplicada.

Bengtsson y Saito (2000).

Martínez Carrión y Moreno Lázaro (2007).

Cámara (2006).

Cámara (2009). 


\section{Nivel de vida biológico durante la consolidación de la sociedad moderna en España}

En paralelo al debate sobre el crecimiento económico y la evolución del sector agrario en España durante el siglo XIX ${ }^{11}$, los estudios sobre nivel de vida realizados en nuestro país durante la última década incorporando variables alternativas como la salud y el nivel educativo, particularmente en el ámbito rural, han contribuido a una visión más compleja del fenómeno. Por ejemplo, han permitido valorar en términos más integrales (más allá del acceso a la propiedad y explotación de la tierra o los niveles de renta) las implicaciones del proceso de crecimiento económico y modernización en España ${ }^{12}$. Sabemos, por ejemplo, que tampoco el campo del bienestar humano fue una balsa de aceite durante el siglo xIX y la primera mitad del Xx. Ciertamente los estudios antropométricos centrados en esos periodos confirman las impresiones que ensayos previos sobre mortalidad y esperanza de vida nos ofrecían: esos aspectos del nivel de vida de la población, particularmente la rural, no presentaron mejoras sustanciales durante la segunda mitad del siglo XIX. Ahora bien, la ausencia de crecimiento sostenido fue compatible con ciclos alternativos de mejora y deterioro, con inestabilidad y con fuertes variaciones interanuales de la estatura como consecuencia de la sucesión de crisis de diversa índole (epidemias o escasez de alimentos, fundamentalmente). En concreto, el periodo 1840-70 parece ser el más crítico y coincide plenamente con tasas muy elevadas de mortalidad infantil y una baja esperanza de vida ${ }^{13}$ así como con el descenso del poder adquisitivo de las clases populares reflejado por la evolución de los salarios reales en el medio rural ${ }^{14}$.

Aunque carecemos por el momento de series continuas de estatura para el conjunto de España durante la segunda mitad del Ochocientos las aproximaciones locales y regionales nos ofrecen un buen contexto de análisis y apuntan a que la estatura media de partida de los españoles hacia mediados del siglo XIX estaba entre las más bajas de los países occidentales (Cuadro 1).

El doctor Olóriz (1855-1912), a partir del estudio de los reclutamientos de 1860-61 (generaciones nacidas en 1840-41) estimaba la talla media de los varones españoles en 1,63 m. Aranzadi y Hoyos lo hacían en 1,62 m aproximadamente ${ }^{15}$. Sobre la información corregida de los Anuarios Estadísticos Españoles, Gómez Mendoza y Pérez Moreda apuntaban que el registro de Olóriz sería válido sólo para los mozos útiles de la época y hacían descender la estatura media masculina a 1,61 m para el reemplazo de 1858 (nacidos en 1838) ${ }^{16}$. Teniendo en cuenta el margen de variación interanual (importante durante la época) y que en dicho reemplazo en concreto no estaban incluidos los mozos redimidos a metálico, creemos que tanto los datos de Olóriz como los de Aranzadi y Hoyos no eran nada aventurados. De hecho, están bastante acordes con los resultados de las series locales y regionales que se aportan en el Cuadro 1 incluso si no estandarizáramos esos datos a la edad de 21 años. Es decir, que probablemente el varón español de mediados del siglo XIX habría medido entre 1,62 y 1,64 m habiendo completado su ciclo de crecimiento. En dos cortes temporales posteriores realizados bajo reemplazos modernos de reclutamiento universal (1913 y 1917, generaciones nacidas en

\footnotetext{
11 cfr. Tortella (1992); Simpson (1995); vs. Bringas Gutiérrez (2000); Pujol et al. (2001); Barciela et al. (2005).

12 Martínez Carrión (2002).

13 Sanz Gimeno y Ramiro Fariñas (2002); Nicolau (2005).

14 Moreno Lázaro (2002, 2006); Lana Berasain (2002).

15 Cit. en Gómez Mendoza y Pérez Moreda (1985), pp. 45-46.

16 Gómez Mendoza y Pérez Moreda (1985).
} 


\section{CUADRO 1}

REFERENCIAS APROXIMADAS DE ESTATURA MEDIA PARA LAS GENERACIONES MASCULINAS NACIDAS EN LA SEGUNDA MITAD DEL SIGLO XIX (MILÍMETROS) $)^{17}$

\begin{tabular}{|c|c|c|c|c|}
\hline País & $\begin{array}{l}\text { Mediados } \\
\text { del siglo XIX }\end{array}$ & España & $\begin{array}{l}\text { Mediados } \\
\text { del siglo XIX }\end{array}$ & $\begin{array}{c}\text { Finales } \\
\text { del siglo XIX }\end{array}$ \\
\hline Estados Unidos & 1.711 & Sureste ${ }^{18}$ & 1.623 & 1.634 \\
\hline Noruega & 1.686 & Levante $^{19}$ & 1.627 & 1.638 \\
\hline Suecia & 1.682 & Granada rural $^{20}$ & 1.631 & 1.632 \\
\hline Austria & 1.672 & Centro $^{21}$ & 1.619 & 1.630 \\
\hline Dinamarca & 1.661 & Castilla y León ${ }^{22}$ & 1.615 & 1.625 \\
\hline Bélgica & 1.655 & Cataluña Occidental ${ }^{23}$ & 1.639 & 1.646 \\
\hline Gran Bretaña & 1.653 & España ${ }^{24}$ & 1.609 & 1.620 \\
\hline Holanda & 1.651 & España $\|^{25}$ & & 1.640 \\
\hline Francia & 1.647 & & & \\
\hline Alemania & 1.643 & & & \\
\hline Italia & 1.622 & & & \\
\hline
\end{tabular}

Fuente: ver notas al pie.

1892 y 1896 que fueron medidas contando un año más, veintiuno) Gómez Mendoza y Pérez Moreda estimaban una estatura media sólo ligeramente por encima de 1,62 m para el conjunto de los quintados y entre 1,63 y 1,64 m para los mozos finalmente declarados útiles ${ }^{26}$. Esto querría decir que en el medio siglo comprendido entre las décadas de 1840 y 1890 la estatura media de los españoles apenas habría aumentado 1 o $2 \mathrm{~cm}$ (eso, dando por buenas las esti-

17 Los datos europeos y norteamericanos proceden de diversos trabajos y fueron recopilados por Crafts (1997), p. 628. Las edades de reclutamiento oscilan entre 20 y 23 años y se refieren a generaciones nacidas en torno a 1850. Las referencias para España se especifican más abajo. Para mediados del siglo XIX corresponden a mozos de 20 años y la estatura que incluimos en el Cuadro es una media aproximada de la presentada por los autores en series de medias móviles quinquenales entre 1850 y 1860, estandarizada a 21 años. Las de finales del XIX corresponden a mozos medidos con 21 años y nacidos entre 1890 y 1900 salvo que se especifique otro dato.

186 localidades de las provincias de Murcia y Alicante. Martínez Carrión y Moreno Lázaro (2007).

1911 localidades de las provincias de Alicante, Valencia y Castellón (1861-65 y 1891-95). Martínez Carrión y Puche Gil (2009).

202 localidades de la provincia de Granada. Cámara (2007).

$21 \quad 18$ localidades de la región de Madrid estandarizadas a 21 años. García Montero (2009).

226 localidades de la región de Castilla y León estandarizadas a 21 años. Martínez Carrión y Moreno Lázaro (2007).

2326 localidades catalanas estandarizadas a 21 años (1846-60. Ramón i Muñoz (2009).

24 Gómez Mendoza y Pérez Moreda (1985), pp. 45-46. Mozos nacidos en 1838 y tallados en 1858 contando 20 años de edad. Talla no estandarizada.

25 Quiroga (2002) a partir de expedientes de soldados centralizados en el Archivo General Militar de Guadalajara. Suponemos que la mayoría de ellos fueron tallados a los 21 años pero sin embargo en la clase tropa de la que proviene la información hemos constatado la ausencia generalizada de mozos declarados cortos de talla y previamente excluidos del reclutamiento por lo que es posible que ese registro para el conjunto nacional esté algo sesgado al alza.

26 Gómez Mendoza y Pérez Moreda (1985), p. 48. 
maciones de partida más pesimistas y teniendo en cuenta que entre los 20 y los 21 años existía aún un margen, aunque probablemente reducido, de crecimiento $\left.{ }^{27}\right)^{28}$. Las series locales elaboradas confirman este extremo. Hacia mediados del siglo XIX el rango de estaturas medias en las áreas analizadas estaba comprendido entre 1.615 y $1.639 \mathrm{~mm}$ aproximadamente $(24 \mathrm{~mm}$ de diferencia entre las regiones con mozos más altos y aquellas con las estaturas medias más bajas) ${ }^{29}$; para las cohortes nacidas hacia finales de esa centuria el rango no sólo se mantenía bastante estable sino que lo hacía atendiendo a unos márgenes de mejora de la estatura media muy escasos en un intervalo temporal de cinco décadas (de $1.625 \mathrm{~mm}$ de los castellano-leoneses a $1.646 \mathrm{~mm}$ de los catalanes occidentales que seguían siendo los más altos entre las áreas peninsulares estudiadas, es decir, $21 \mathrm{~mm}$ de diferencia). En este rango regional, series como la levantina o la granadina, presentando valores intermedios, pueden constituir laboratorios válidos de referencia para ensayar la prolongación de las series y extrapolar resultados a ámbitos más amplios. En este caso hemos trabajado con los datos de las dos localidades que aparecen como representativas de la Granada Rural. Puede observarse que las medias registradas a mediados del siglo XIX, en torno a 1,63 m están entre los valores extremos mostrados por Castilla-León y Cataluña. A finales del siglo XIX, dado el poco progreso mostrado por las localidades andaluzas, sus valores medios pueden ya considerarse bajos en el contexto de las áreas analizadas pero aún representativos en el contexto español puesto que el progreso de las áreas levantinas, incluida Cataluña, es bastante relevante y hasta cierto punto excepcional dentro del panorama antropométrico peninsular.

\section{Fuentes y metodología}

\subsection{Localidades de estudio}

Los datos sobre estatura que se presentan pertenecen a las localidades andaluzas de Montefrío y Santa Fe, representativas de contextos ambientales y socioeconómicos diferenciados.

Montefrío es una comunidad de media montaña situada a unos $40 \mathrm{~km}$ al noroeste de la ciudad de Granada y de limitada accesibilidad aún en nuestros días. Santa Fe es una población situada en el valle del río Genil, en el área metropolitana de la capital granadina. El municipio de Montefrío a mediados del siglo XVIII tenía alrededor de 5.000 habitantes mientras que el de Santa Fe estaba en torno a los 2.500. Un siglo después, hacia 1850 esas poblaciones eran de 8.000 y 4.700 habitantes respectivamente. Montefrío superó los 10.000 habitantes en 1877 mientras que Santa Fe se mantuvo en torno a los 5.000 hasta finales del siglo XIX si bien el hábitat en Montefrío era mucho más disperso que en Santa Fe, cuyo núcleo urbano concentraba a la mayoría de esos habitantes.

Estas comunidades han sido seleccionadas porque sobre ellas se han realizado previamente diversos trabajos de historia agraria que aportan información precisa sobre producción, sistemas de propiedad y, en general, sobre las dinámicas concretas que la transición

27 Sirva como referente comparativo un intervalo temporal equivalente un siglo después: entre 1940 y 1980 la estatura media declarada de los varones españoles mostró un progreso de casi $8 \mathrm{~cm}$. Spijker et al. (2008).

28 Martínez Carrión y Pérez Castejón (2000); Cámara (2007b).

29 Nótese que ese rango es más amplio cuando se consideran en detalle las distintas series locales dentro de cada área. 
entre el Antiguo Régimen y la sociedad moderna revistió en aquella zona ${ }^{30}$. Por tanto, ofrecen un escenario socioeconómico muy bien estudiado sobre el que poder realizar ulteriores interpretaciones de los resultados aportados por indicadores alternativos de nivel de vida como la estatura. A grandes rasgos, según los trabajos citados, las privilegiadas condiciones ambientales de la vega de Granada, particularmente la abundancia de agua, favorecieron una especialización temprana en cultivos de regadío orientados a la comercialización. El predominio del suelo agrícola y las formas modernas de propiedad dan testimonio de una temprana transición al capitalismo agrario en Santa Fe desde el último tercio del siglo XVIII. En los montes granadinos los sistemas de aprovechamiento mantuvieron durante algún tiempo más su carácter integrado, con mucha importancia de los terrenos comunales que sostuvieron una importante cabaña ganadera. De ahí que, presumiblemente, la economía de Montefrío basculase más hacia el autoconsumo al menos hasta mediados del siglo XIX.

\subsection{Descripción de los datos antropométricos}

Los datos antropométricos de Montefrío proceden de las Actas de Declaración y Clasificación de Soldados y Suplentes (ASS). Los de Santa Fe proceden de dos fuentes: las mencionadas actas y los Libros de Filiación provinciales (LF) que desde el último tercio del siglo XIX se basaron en el mismo criterio de registro que las actas locales ${ }^{31}$. La problemática de estas fuentes se resume en los siguientes aspectos.

Los datos antropométricos de la segunda mitad del siglo XIX (pertenecientes a las cohortes nacidas a partir de 1838) son fácilmente integrables en series temporales ya que se homologa a una edad única el reclutamiento para cada quinta y se expresa la talla en milímetros de todos los mozos (útiles, exceptuados y excluidos). Para periodos anteriores esto no es así. En primer lugar, antes de 1850 encontramos a mozos de varias edades incluidos en un mismo alistamiento. En segundo lugar, aunque en muchos alistamientos se medía a todos los mozos (adelantamos que en este trabajo sólo se utilizan datos de este tipo de alistamientos universales), el registro de las tallas en las actas no es exhaustivo. La diferencia de las denominadas muestras truncadas con respecto a este tipo de muestras es que aunque aquí no se dispone de las tallas de los excluidos sí se conoce cuántos y quiénes eran, lo cual es una ventaja con respecto a los registros militares truncados propiamente dichos en los que sólo se incluye a los individuos que finalmente integraron el cupo de soldados de un ejército (este tipo de registro también puede encontrarse en España pero es minoritario con respecto al registro universal). A efectos prácticos, no obstante, el problema metodológico es el mismo que presentan las muestras truncadas, es decir, estimar correctamente la estatura del conjunto de la población de la cual procede la muestra (sesgada a la derecha en el caso de los cupos de soldados).

Digamos, por tanto, que en el caso español el problema para muchos alistamientos de la primera mitad del siglo XIX es que contamos con las tallas de los individuos que estaban por encima del mínimo establecido mientras que el resto de tallas, o bien no aparece o aparece bajo anotaciones muy genéricas como "no marca". En estos casos, puesto que se conoce una parte de la distribución de estaturas y dado que esa distribución sigue el modelo gaussiano o normal puede estimarse la otra parte de la distribución a través de técnicas de regresión truncada ${ }^{32} \mathrm{o}$

30 Gómez Oliver y González de Molina (1983); Martínez Martín (1995); Martínez López (1996); González de Molina y Guzmán Casado (2006).

31 Cámara (2006).

32 A'Hearn (2004); Komlos (2004); Challú (2009). 
imputación de la estatura en función de otras covariables observadas a nivel individual ${ }^{33}$. Por tanto, contar con tendencias basadas en series de estatura en España desde principios del siglo XIX es factible, pero no es el objetivo de este trabajo. Aquí lo que pretendemos es comenzar a averiguar si las estaturas del Antiguo Régimen fueron superiores a las de las primeras fases de la modernización. Y en el caso de los alistamientos del siglo XVIII el problema que plantean las fuentes es distinto a todo lo expuesto hasta ahora. Esos alistamientos del Setecientos siguen siendo universales dando lugar, estrictamente, a distribuciones de estatura no truncadas. Ahora bien, el inconveniente es que por lo general sólo se dispone de una anotación indicando, para cada mozo alistado, si se estaba por encima o por debajo de un mínimo establecido (que en este caso era de cinco pies franceses o $1.625 \mathrm{~mm}$ ). Es decir, no sólo ignoramos las estaturas exactas de la cola izquierda de la distribución (los excluidos del servicio militar por no dar la talla) sino también las de la cola derecha (los mozos declarados útiles). Este sistema de registro dificulta la elaboración de series temporales de estaturas medias por cohorte de nacimiento (aproximación clásica de la Antropometría Histórica) así como la aplicación de metodologías de estimación sobre datos truncados como las citadas. En la práctica, nuestra variable dependiente (la estatura) adquiere, por tanto, una naturaleza dicotómica. Y puesto que sólo conocemos los mínimos exigidos y la referencia de si un mozo estaba por encima o por debajo, hemos procedido aplicando una técnica como la regresión logística que, a través de una variable dependiente dicotómica (marcar o no marcar ese mínimo requerido) nos informará de la variación del riesgo de caer en un determinado rango de estaturas (en este caso $1.625 \mathrm{~mm}$ o más) a lo largo del tiempo. Es decir, que hemos transformado toda nuestra muestra de estaturas en valores discretos $(0,1)$ para homologar todos los datos al sistema de registro del siglo XVIII. Dado que el mínimo requerido es variable en el tiempo, se procede homologando el mismo al más alto histórico dentro del periodo analizado ${ }^{34}$ (esto es, $1.625 \mathrm{~mm}$ en el caso de las tropas regulares españolas que son de las que provienen nuestros datos ${ }^{35}$ ). Ofrecemos más detalles sobre la construcción de la variable y el modelo en el apartado 3.3.

La principal limitación de esta metodología, forzada por la naturaleza de la información contenida en las fuentes, es que no controla los cambios que pueden darse en un determinado segmento de la distribución de estaturas. Esto puede provocar que las variaciones en la estatura media no respondan a una evolución paralela de, por ejemplo, los distintos cuartiles de la distribución, sino a las variaciones del cuartil superior o inferior, por poner el caso. Dicho de otro modo, esta técnica no proporciona información sobre el conjunto de la distribución de estaturas y podría ocurrir, por ejemplo, que un ciclo de descenso de la estatura media, con respecto al punto de truncamiento establecido $(1.625 \mathrm{~mm})$ se estuviera debiendo al hundimiento de un segmento de la distribución mientras que el resto de segmentos estuvieran progresando positivamente. En tal caso, por consiguiente, la información proporcionada por el modelo sería parcial o poco representativa del conjunto de la población. Hay dos elementos, a juicio de los especialistas, que hacen improbable que eso ocurra: la normalidad de la distribución de estatu$\operatorname{ras}^{36}$ y la estabilidad y reducido rango de la dispersión de estaturas con respecto a la media ${ }^{37}$.

En la Figura 1 se presentan las distribuciones de la variable, estandarizada a 21 años (puede verse al respecto el apartado 3.4), para cuatro cortes temporales que agrupan cinco cohortes cada uno. En este caso, puesto que el alistamiento de 1808 de la localidad de Mon-

\footnotetext{
33 Rubin (1987); Quaranta (2007).

34 Komlos y Kim (1990).

35 Cámara (2006).

36 Tanner (1986); Bogin (1988).

37 Cole (2000, 2003); A'Hearn (2004).
} 
tefrío registró exhaustivamente las tallas de todos los mozos, los análisis incluyen un grupo de cohortes nacidas durante el último tercio del siglo XVIII compuestas por varones medidos con 19, 20, 21, 22 y 23 años. Esas distribuciones muestran una normalidad bastante aceptable (pueden observarse claramente los efectos del redondeo en torno a determinados registros, particularmente cuando la talla no era expresada en milímetros en las fuentes, pero a pesar de todo las distribuciones responden claramente a reclutamientos con carácter universal en cuanto que las colas de la distribución no aparecen erosionadas significativamente ${ }^{38}$.

\section{FIGURA 1}

ESTADÍSTICOS BÁSICOS, DISTRIBUCIONES DE FRECUENCIAS Y CURVA NORMAL PARA CUATRO SUBMUESTRAS DE DATOS (ESTATURAS EN MILÍMETROS)

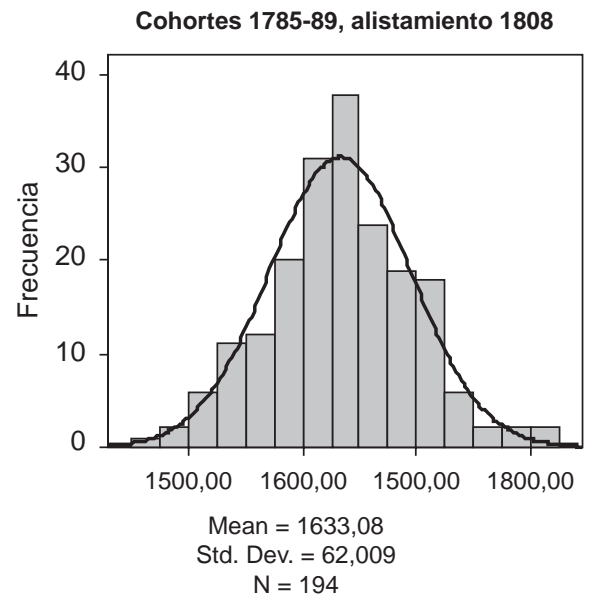

Cohortes 1887-91, alistamientos 1908-12

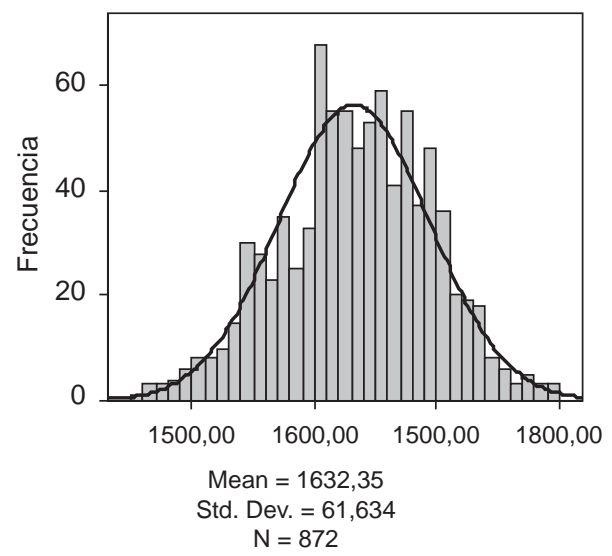

Fuente: Elaboración propia a partir de ASS y LF.
Cohortes 1838-42, alistamientos 1858-62

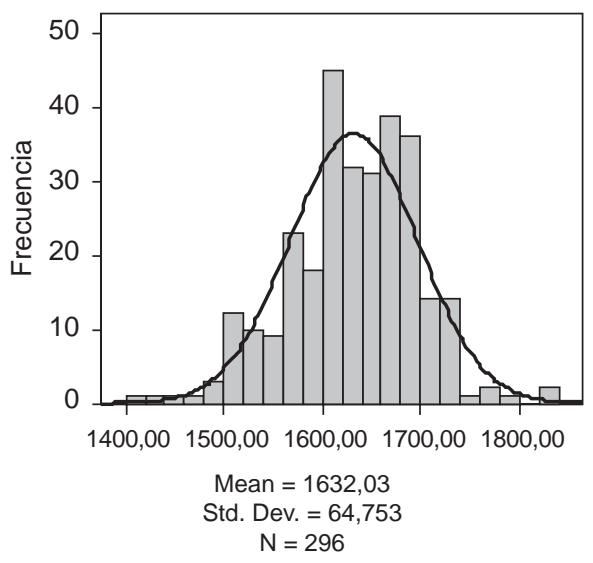

Cohortes 1928-32, alistamientos 1949-53

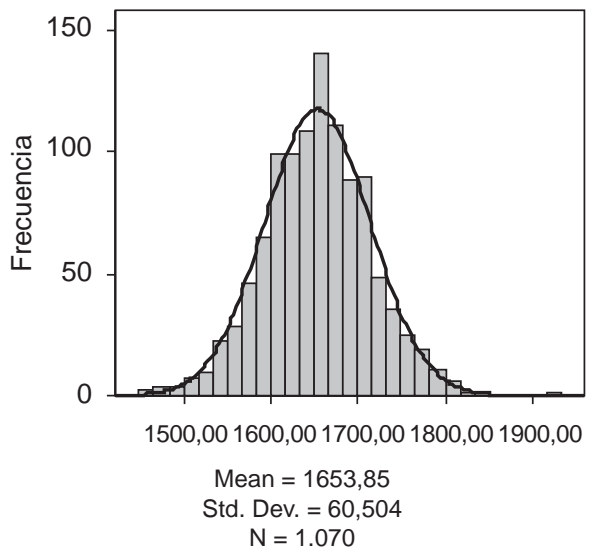

$38 \quad$ Komlos (2004). 
Otro asunto es la representatividad específica de la media en nuestra distribución (lo que implica cuestionarse la validez de las técnicas basadas en este estadístico como la regresión basada en mínimos cuadrados, Ordinary Least Squares, OLS, o la basada en la estimación de la probabilidad máxima, Maximum Likelihood Estimation, $\left.M L E^{39}\right)$. En este punto hay que decir que es imposible indagar en la evolución de esa distribución de estaturas durante el siglo XVIII fuera de los datos que ofrece el alistamiento de 1808 en Montefrío. En cambio, a través de los datos de los siglos XIX y XX, sí podemos valorar hasta qué punto es asumible el riesgo de trabajar con una variable dicotómica que informa sobre tendencias a largo plazo clasificando a los individuos sólo en dos categorías (por encima o por debajo de $1.625 \mathrm{~mm}$ ).

En el Gráfico 1 presentamos la evolución anual de los cuartiles de la distribución de estatura por cohortes de nacimiento para las dos localidades estudiadas en la que incluso teniendo en cuenta la dimensión anual puede apreciarse el notable paralelismo de esos tres segmentos de la distribución (más adelante, el Gráfico 3 ofrece la evolución de las medias móviles trienales de los datos de la muestra agregados en grupos que contienen el $25 \%$ de los casos lográndose, lógicamente, mayor uniformidad en los resultados). De manera destacable, el registro que utilizamos para el diseño de la variable dicotómica $(1.625 \mathrm{~mm})$ sólo se localiza en el cuartil inferior para cohortes de nacimiento muy concretas y ya en el siglo xx. Esto quiere decir que la incidencia de los valores de la variable dicotómica (marcar o no marcar $1.625 \mathrm{~mm}$ ) no está determinada por valores extremadamente altos o bajos, sino que tanto en el segmento superior como en el inferior de la distribución existe un buen rango de valores que amortiguan ese posible sesgo.

GRÁFICO 1

DISTRIBUCIÓN DE LOS CUARTILES DE ESTATURA POR COHORTE

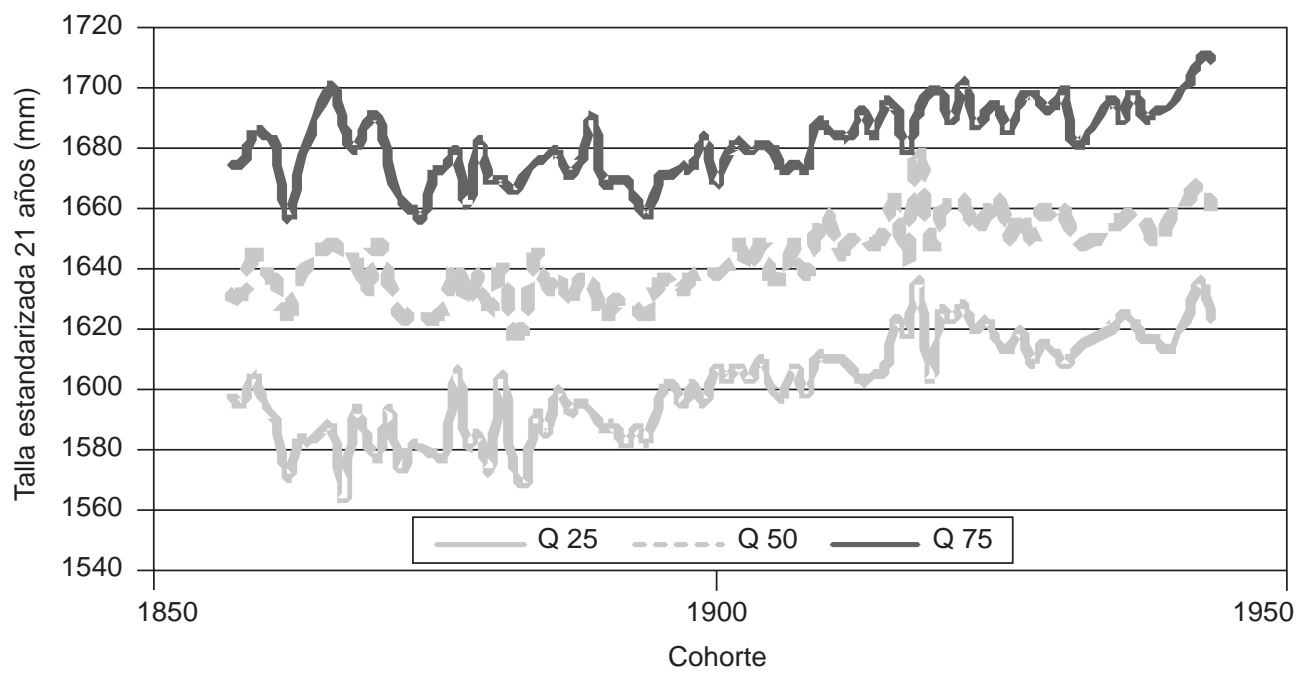

Fuente: Elaboración propia a partir de ASS y LF.

39 Ambas técnicas, la primera bajo la variante TOLS (Truncated Ordinary Least Squares), han sido preferidas desde hace más de una década a la primera técnica propuesta por Wachter y Trusell (1982), el Quantile Bend Estimator. Al respecto puede verse Komlos (2004). 
Por último, el Cuadro 2 presenta la evolución de la media de estatura y de la desviación típica para las dos localidades de las que proceden nuestros datos. El grado de coherencia con lo teorizado previamente acerca del comportamiento de la variable estatura es altamente satisfactorio. Constatamos que el rango de la desviación típica entre diversas poblaciones o en una población a lo largo del tiempo siempre se mueve en márgenes muy estrechos incluso cuando las diferencias en las medias respectivas (entre dichas poblaciones o en el tiempo) puedan alcanzar rangos muy amplios ${ }^{40}$. Podemos observar que las desviaciones con respecto a la media son superiores en periodos donde desciende la estatura y, si bien a largo plazo se da un proceso de concentración de las estaturas (disminuye la desviación típica), podemos comprobar que ni el proceso es lineal ni es probable que las desviaciones típicas anteriores a las cohortes nacidas a mediados del siglo XIX fuesen superiores a las alcanzadas en esa época a tenor de la evidencia empírica aportada por otros trabajos así como por nuestra muestra procedente del alistamiento de 1808. En cualquier caso, mediante una agregación de cohortes tan amplia como la que se propone en el siguiente apartado (cincuenta años) se incluyen ciclos cortos de descenso y recuperación de la estatura y, por tanto, una desviación típica muy suavizada y estable para los cuatro grupos de cohortes sobre los que basaremos el análisis.

CUADRO 2

ESTADÍSTICOS BÁSICOS PARA AMBOS PUEBLOS DESDE 1857 EN ADELANTE

\begin{tabular}{lrrrrrrrrr}
\hline & \multicolumn{3}{c}{ Montefrío } & \multicolumn{3}{c}{ Santa Fe } & \multicolumn{3}{c}{ Total } \\
\cline { 2 - 10 } Cohorte & Media & N & $\begin{array}{c}\text { Desv. } \\
\text { Típica }\end{array}$ & Media & N & $\begin{array}{c}\text { Desv. } \\
\text { Típica }\end{array}$ & Media & N & $\begin{array}{c}\text { Desv. } \\
\text { Típica }\end{array}$ \\
\hline $\mathbf{1 8 5 7 - 1 8 6 6}$ & $1.639,8$ & 856 & 69,8 & $1.621,4$ & 404 & 73,7 & $1.633,9$ & 1.260 & 71,5 \\
$\mathbf{1 8 6 7 - 1 8 7 6}$ & $1.631,4$ & 1.019 & 73,8 & $1.618,9$ & 488 & 72,3 & $1.627,4$ & 1.507 & 73,5 \\
$\mathbf{1 8 7 7 - 1 8 8 6}$ & $1.628,1$ & 1.013 & 68,1 & $1.635,9$ & 550 & 63,9 & $1.630,9$ & 1.563 & 66,7 \\
$\mathbf{1 8 8 7 - 1 8 9 6}$ & $1.631,4$ & 1.187 & 61,9 & $1.631,6$ & 319 & 54,5 & $1.631,4$ & 1.506 & 60,4 \\
$\mathbf{1 8 9 7 - 1 9 0 6}$ & $1.640,6$ & 976 & 60,2 & $1.640,1$ & 693 & 60,8 & $1.640,4$ & 1.669 & 60,4 \\
$\mathbf{1 9 0 7 - 1 9 1 6}$ & $1.643,9$ & 1.106 & 59,8 & $1.656,2$ & 784 & 65,1 & $1.649,0$ & 1.890 & 62,3 \\
$\mathbf{1 9 1 7 - 1 9 2 6}$ & $1.658,7$ & 1.092 & 58,4 & $1.657,1$ & 429 & 54,5 & $1.658,2$ & 1.521 & 57,3 \\
$\mathbf{1 9 2 7 - 1 9 3 6}$ & $1.655,3$ & 1.330 & 60,0 & $1.657,0$ & 619 & 58,5 & $1.655,9$ & 1.949 & 59,5 \\
$\mathbf{1 9 3 7 - 1 9 4 6}$ & $1.661,0$ & 908 & 60,2 & $1.667,4$ & 554 & 57,4 & $1.663,5$ & 1.462 & 59,2 \\
\hline
\end{tabular}

Fuente: elaboración propia a partir de ASS y LF.

En conjunto, estos análisis previos muestran cambios relativamente reducidos en la dispersión de los datos con respecto a la media (menores incluso cuando se agregan los datos de ambas comunidades y se procede a través de agrupaciones de cohortes más amplias) así como un grado aceptable de normalidad que, comprensiblemente, aumenta cuando son incluidos en el análisis todos los alistamientos disponibles. Por tanto, ante la pregunta de si la evolución de los segmentos de la distribución pudo ser diferente para las cohortes nacidas a mediados del siglo XVIII para las que no disponemos de ninguna información, nuestra respuesta es, obligatoriamente, sí. Ante la pregunta de si esto es probable teniendo en cuenta la 
evolución de la distribución durante más de cien años, la respuesta es no y por eso creemos que la aplicación de la regresión logística para el análisis conjunto de todos los datos antropométricos disponibles desde el siglo XVIII al XX es una metodología válida de acuerdo con el objetivo inicial del trabajo (recuérdese que lo que nos preocupa es saber si las diferencias observadas en el tiempo con respecto a un criterio constante son significativas $y$, segundo, que al menos el signo de las tendencias obtenidas sea fiable).

Esta aproximación econométrica se complementa con otras dos: la construcción de una serie temporal de estatura desde mediados del siglo XIX (entonces ya con registros de estatura específicos) y con otra aproximación de tipo microhistórico basada en la selección de los jóvenes pertenecientes a familias de grandes labradores y propietarios de la comunidad de Montefrío para su comparación con el conjunto de la comunidad.

\subsection{Elaboración del modelo de regresión logística}

El modelo se basa en una agrupación de cohortes suficientemente amplia para obtener tendencias a largo plazo que sean significativas desde el punto de vista estadístico. Para el análisis sólo se han utilizado datos de aquellas actas correspondientes a reclutamientos en los que la medición de los quintos fue previa al juicio de exenciones y al sorteo (alistamientos universales). Así nos aseguramos que están incluidas las estaturas (o sus referencias indirectas) de todos los mozos alistados, tanto los declarados útiles como los que finalmente quedaron exceptuados o excluidos del servicio militar por distintos motivos. Ese requisito, así como que el reemplazo aporte datos directos o indirectos sobre estatura, es cumplido por los alistamientos locales de 1771, 1773, 1775, 1794, 1808, 1809, 1823, 1824 y a partir de 1836. A pesar de que los datos corresponden a mozos tallados a distintas edades, no se realiza ninguna estandarización puesto que la edad es incluida como variable de control en el modelo. Lo que sí se estandariza es el mínimo de estatura exigido que fue cambiando en la legislación sobre quintas desde el último tercio del siglo XVIII. Como se mencionaba más arriba, hemos fijado un listón constante de $1.625 \mathrm{~mm}$ que corresponde al mínimo exigido en los reemplazos más antiguos disponibles para las comunidades de estudio (además es el máximo histórico establecido para las tropas regulares en el reclutamiento por quintas desde 1741). Con estas premisas se ha creado una variable dicotómica que expresa la cualidad de marcar o no marcar el mencionado registro (0 No marca; 1 Marca) y que actuará como variable dependiente en el modelo (Cuadro 3).

CUADRO 3

ANÁLISIS DE CASOS PARA LA VARIABLE DICOTÓMICA

\begin{tabular}{llccc}
\hline & & Frecuencia & $\%$ & $\%$ válido \\
\hline Válidos & No marca 1625 & 6.877 & 34,6 & 39,5 \\
& Marca 1625 & 10.552 & 53,1 & 60,5 \\
\cline { 2 - 5 } & Total & 17.429 & 87,7 & 100,0 \\
\hline Perdidos & 2.436 & 12,3 & \\
\hline Total & & 19.865 & 100,00 & \\
\hline
\end{tabular}


Hubo cambios en ese mínimo exigido. Cuando la talla viene expresada numéricamente para todos los alistados este hecho no implica problemas. Pero cuando las referencias de talla son indirectas se crean algunos inconvenientes. Por ejemplo, hasta que el registro no fue exhaustivo y numérico, se dieron dos cambios en la legislación y el mínimo exigido descendió a 1.597 y $1.582 \mathrm{~mm}$ en determinados alistamientos (Cuadro 4). Mientras que podíamos asegurar que los quintos que no marcaban podían agruparse en la categoría 0, aquellos que daban la talla podrían haber llegado o no a los consabidos $1.625 \mathrm{~mm}$. De incluir sólo a los primeros en el análisis hubiera resultado un sesgo a la baja en la cohorte de referencia del modelo (generaciones nacidas antes de 1800) puesto que el mínimo de 1.582 fue el exigido en el alistamiento de 1809. Puesto que se disponía de todas las tallas expresadas en milímetros para el gran alistamiento de 1808 en Montefrío, se optó directamente por descartar 1809. El mismo problema se planteaba con el mínimo de $1.597 \mathrm{~mm}$ pero en este caso sólo fue necesario descartar los alistamientos de 1845 y 1846 puesto que a partir de 1837, salvo en esos dos años, contábamos con las tallas de los mozos que sí llegaron a ese mínimo. En cualquier caso, realizamos pruebas incluyendo en el modelo dentro de la categoría 0 tanto los mozos que no marcaron 1.682 como aquellos que no llegaron a 1.597 y no hubo cambios sustanciales de tendencia ni de intensidad.

\section{CUADRO 4}

EVOLUCIÓN DE LA ESTATURA MÍNIMA EXIGIDA EN ESPAÑA PARA INGRESAR EN EL EJÉRCITO, 1741-1912 (TROPAS REGULARES DE REEMPLAZO)

\begin{tabular}{llc}
\hline & Medida original citada en las fuentes & Conversión (mm) \\
\hline $\mathbf{1 7 4 1}$ & Cinco pies (Pie de Roi o de París) & 1.625 \\
$\mathbf{1 7 6 2}$ & Cinco pies y una pulgada & 1.656 \\
$\mathbf{1 7 7 0}$ & Cinco pies & 1.625 \\
$\mathbf{1 8 0 8}$ & Cinco pies menos una pulgada & 1.597 \\
$\mathbf{1 8 0 9}$ & Cinco pies menos una pulgada y media & 1.582 \\
$\mathbf{1 8 1 9}$ & Cinco pies menos media pulgada & 1.609 \\
$\mathbf{1 8 2 1}$ & Cinco pies menos una pulgada & 1.597 \\
$\mathbf{1 8 3 6}$ & Cuatro pies, diez pulgadas y seis líneas & 1.582 \\
$\mathbf{1 8 3 7}$ & Cinco pies menos una pulgada & 1.597 \\
$\mathbf{1 8 5 6}$ & Cinco pies (Marco de Burgos) & 1.596 \\
$\mathbf{1 8 5 9}$ & $1.569 \mathrm{~mm}$ & 1.569 \\
$\mathbf{1 8 6 0}$ & $1.560 \mathrm{~mm}$ & 1.560 \\
$\mathbf{1 8 7 5}$ (segundo reemplazo) & $1.530 \mathrm{~mm}$ & 1.530 \\
$\mathbf{1 8 7 7}$ & $1.540 \mathrm{~mm}$ & 1.540 \\
$\mathbf{1 8 8 2}$ & $1.545 \mathrm{~mm}$ & 1.545 \\
$\mathbf{1 9 1 2}$ & $1.540 \mathrm{~mm}$ & 1.540 \\
\hline
\end{tabular}

Fuente: elaboración propia a partir de las siguientes fuentes: Siglo XVIII: 1741, J.A. Portugués (1764); 1762 y 1770 en Gómez Ruiz y Alonso Juanola (1991), pp. 593-594. Siglo XIX: 1809, Circular de la Junta Superior de Gobierno del Reino de Granada a las justicias de los pueblos en 28 de noviembre de 1809 (Archivo Histórico Municipal de Montefrío, Seción 5.8 Servicios Militares, legajo 1773-1821/22); 1821, Circular número 52 (misma signatura); 1819, 1859 y 1860 en Jiménez Guerrero, (2001), pp. 232 y 234); 1912, Abella (1915). Las referencias restantes, Feijoo (1996), p. 36 y pp. $222-223$. Todas las conversiones a milímetros, a partir de Cámara (2006).

Como variable independiente del modelo se ha establecido la cohorte de nacimiento del individuo que ha sido asignada a partir de la edad y la fecha de tallado. En total hemos defi- 
nido tres variables categóricas dicotómicas correspondientes a las agrupaciones de cohortes 1800-49, 1850-99 y nacidos en 1900 o después. De ese modo, las cohortes nacidas antes de 1800 sirven como referencia para comparar la evolución de las probabilidades de marcar a lo largo de periodos sucesivos. Lógicamente, ese grupo de cohortes de referencia no se incluye en el modelo para evitar el efecto de colinearidad perfecta en los análisis.

Las cohortes intermedias se han agrupado en periodos cerrados de 50 años para el siglo XIX puesto que lo que nos interesa constatar son ciclos de estatura a largo plazo y además esta agregación dota al modelo de mayor robustez estadística (Cuadro 5). Las pruebas que realizamos con agrupaciones de 25 años no alteraban ninguna de las tendencias que se observarán en los resultados pero resultaban en menor significatividad estadística. Las agrupaciones de cohortes que delimitan el periodo de estudio son abiertas y por tanto no tienen una amplitud exacta de 50 años. Las cohortes nacidas antes de 1800 incluyen individuos nacidos desde 1735 mientras que las nacidas a partir de 1900 llegan hasta 1943 (es decir, mozos que fueron tallados con 21 años en el año 1964) $)^{41}$.

CUADRO 5

DISTRIBUCIÓN DE LAS AGRUPACIONES DE COHORTES CON RESPECTO A LA VARIABLE DICOTÓMICA

\begin{tabular}{|c|c|c|c|c|c|}
\hline & & & & arca & \\
\hline & & & No marca 1625 & Marca 1625 & Total \\
\hline & Antes de 1800 & Casos & 480 & 737 & 1.217 \\
\hline & & $\%$ & 39,4 & 60,6 & \\
\hline 总 & $1800-1849$ & Casos & 682 & 852 & 1.534 \\
\hline むั & & $\%$ & 44,5 & 55,5 & \\
\hline$\stackrel{g}{ \pm}$ & $1850-1899$ & Casos & 3.249 & 3571 & 6.820 \\
\hline$\stackrel{ }{\frac{}{0}}$ & & $\%$ & 47,6 & 52,4 & \\
\hline & A partir de 1900 & Casos & 2.466 & 5.392 & 7.858 \\
\hline & & $\%$ & 31,4 & 68,6 & \\
\hline & Total & Casos & 6.877 & 10.552 & 17.429 \\
\hline & & $\%$ & 39,5 & 60,5 & \\
\hline
\end{tabular}

Finalmente, la edad a la que fue tallado cada mozo así como el municipio de residencia se incluyen en el modelo como variables de control. Por lo que respecta a la primera, el rango de edades del reclutamiento entre el siglo XVIII y el XX es bastante amplio e incluye algunas en las que el ciclo de crecimiento probablemente no había finalizado (este sería el caso de la gran mayoría de mozos tallados con menos de 21 años). Controlar por la edad nos permitirá centrar el análisis en los efectos generacionales o, dicho de otro modo, aislaremos los efectos

41 Los datos incluidos en la primera agrupación abierta van de 1735 a 1799 con la siguiente distribución por periodos: 1735-49 (277 casos); 1750-74 (519 casos); 1775-99 (421 casos). 
de edad de los efectos de generación sobre la estatura, haciéndolos independientes. Así, el modelo nos permitirá observar si el pertenecer a una determinada agrupación de cohortes influye significativamente en el riesgo de alcanzar la estatura fijada. Pero además, puesto que algunas edades no están representadas en todas las agrupaciones de cohortes, se ha optado por incluir en el modelo sólo a los mozos mayores de 18 años (Cuadro 6). La edad también se ha incorporado en el modelo como variable categórica, asumiendo así que su efecto sobre la estatura no es lineal. De este modo, se han creado dos variables dicotómicas para las edades 20 y $21+$ quedando la edad 19 como referencia.

CUADRO 6

AGRUPACIÓN DE COHORTES Y RANGO DE EDADES ${ }^{42}$

\begin{tabular}{cccccc}
\hline \multirow{2}{*}{ Edad de tallado } & \multicolumn{6}{c}{ Cohortes } & \multirow{2}{*}{ Total } \\
\cline { 2 - 5 } & Antes de $\mathbf{1 8 0 0}$ & $\mathbf{1 8 0 0 - 4 9}$ & $\mathbf{1 8 5 0 - 9 9}$ & $\mathbf{1 9 0 0}$ y posterior & \\
\hline $\mathbf{1 6}$ & 183 & 3 & 0 & 0 & 186 \\
$\mathbf{1 7}$ & 316 & 13 & 0 & 0 & 329 \\
$\mathbf{1 8}$ & 224 & 437 & 0 & 0 & 661 \\
\hline $\mathbf{1 9}$ & 186 & 510 & 2.558 & 0 & 3.254 \\
$\mathbf{2 0}$ & 199 & 1.075 & 2.466 & 1.010 & 4.750 \\
$\mathbf{2 1 +}$ & 1.033 & 234 & 2.332 & 8.620 & 12.219 \\
\hline Total & 2.141 & 2.272 & 7.356 & 9.630 & 21.399 \\
\hline Total 19+ & 1.418 & 1.819 & 7.356 & 9.630 & 20.223 \\
\hline
\end{tabular}

El municipio de alistamiento se incorpora como variable de control para constatar que las posibles diferencias observadas entre las cohortes agregadas no responden a ciclos diferenciados en la evolución del estado nutricional en ambas comunidades que, por otra parte, se dieron en el corto plazo (Cuadro 2$)^{43}$.

Como puede intuirse, estamos disponiendo nuestros datos bajo una estructura en la que desconocemos la distribución subyacente de probabilidades y sólo podemos medir si la probabilidad es igual a 0 o igual a 1. También asumimos que esa distribución probabilística no es linear ya que ninguna de las variables independientes incluidas produce incrementos lineales en la estatura. Es más, puesto que las funciones probabilísticas suelen ser s-shaped, la función logística es la opción que hemos elegido para llevar a cabo la estimación. El resto de parámetros de nuestra ecuación son calculados en función de ese criterio que imponemos (valores predichos de la variable dependiente, entre 0 y 1 ). Podemos resumir esta argumentación con la siguiente notación:

$$
Y=a+b_{1} x_{1}+b_{2} x_{2}+b_{3} x_{3}+b_{4} x_{4}+b_{5} x_{5}+b_{6} x_{6}+e
$$

42 El total para las edades 19+ en cada agrupación de cohortes no coincide con el total de datos válidos para cada agrupación (Cuadro 3) debido al descarte de individuos para los que no se registra ninguna indicación de estatura así como aquellos que fueron quintados en reclutamientos que no cumplen los requisitos expresados para incluir un alistamiento en el análisis. En total se descartan del análisis unos 3.000 casos.

43 Cámara (2007). 
Donde $Y=\log$ (Probabilidad de marcar/Probabilidad de no marcar).

$X_{1}=$ grupo de cohortes $1800-49$

$X_{2}=$ grupo de cohortes 1850-99

$X_{3}=$ grupo de cohortes nacidas en $1900 \mathrm{y}$ posterior

$X_{4}=$ edad 20

$X_{5}=$ edad $21+$

$X_{6}=$ municipio de Santa Fe

Es decir, queremos estimar el impacto de la cohorte de nacimiento en la probabilidad de marcar $1.625 \mathrm{~mm}$ controlando por la edad y el municipio de residencia y sólo disponiendo de la información de si el individuo llegó o no llegó a la marca.

En total, tras los descartes mencionados así como el de aquellos mozos que no pudieron asignarse a una cohorte por no aparecer su edad, los casos incluidos en el análisis ascienden a cerca de 17.500 .

\subsection{Construcción de la serie temporal}

Para la serie temporal de estatura sólo se utilizan aquellos reemplazos con expresión específica de la talla de alistados válidos y excluidos. Aunque para Montefrio se dispone de datos de este tipo desde la década de 1830 hemos preferido comenzar la serie en 1857 que es el primer año para el que se dispone de información sistemática de este tipo para los mozos nacidos en Santa Fe. De este modo, la serie que se presenta es una media de los registros de las dos comunidades e incluye a modos tallados entre los 19 y los 21 años. Para corregir este sesgo, la serie está estandarizada a la edad de 21 años (la máxima exigida en determinados periodos del reclutamiento militar español moderno). Esa estandarización se realiza por la adición de algunos milímetros a los mozos de 19 y 20 años según lo que pudo ser el patrón de crecimiento para dicho periodo que, no obstante, resulta de una estimación imperfecta ya que el seguimiento de mozos es limitado y la regresión edad-estatura sobre la que se plantea la estandarización se basa fundamentalmente en observaciones transversales ${ }^{44}$. Puesto que el modelo de regresión se planteó con las estaturas del centro de la distribución (excluyendo los extremos más alto y bajo respectivamente), en cierto modo se corrige el efecto de sobreestimación del crecimiento potencial adjudicado a los mozos más altos y mejor nutridos que probablemente ya habían completado su ciclo de crecimiento físico a los 19 años o incluso antes. Además, las adiciones resultantes que representan el crecimiento potencial hasta los 21 años $(1,5 \mathrm{~cm}$ para los mozos de 19 años y 0,7 cm para los de 20) se ajustan muy bien a los seguimientos individuales que hemos podido realizar en los casos en que un mozo era tallado en años sucesivos por haber quedado su caso pendiente de resolución. Resultados muy parecidos de estandarización a partir de otros métodos de estimación pueden encontrarse en trabajos previos ${ }^{45}$.

\subsection{Aproximación nominal}

Por último incluimos un ejemplo de las posibilidades que tiene el análisis de los datos antropométricos desde un punto de vista nominal integrándose con información proveniente de otras fuentes. Se presenta la estatura de mozos pertenecientes a cuatro destacadas familias de

44 Cámara (2007), pp. 41 y ss.

45 Martínez Carrión y Moreno Lázaro (2007); Ramón i Muñoz (2009). 
la localidad de Montefrío que tanto en las fuentes fiscales como en las actas capitulares aparecen como grandes propietarias o arrendatarias de tierras así como detentadoras de importantes cargos de la administración municipal. La inclusión de un mozo en esa lista viene determinada por la aparición de alguno de los apellidos seleccionados, en primer o en segundo lugar, en el acta de Declaración correspondiente.

\section{Resultados}

El modelo de regresión resultante (Cuadro 7), tomando como cohortes de referencia la agrupación de las anteriores a 1800 muestra un descenso en la probabilidad de marcar $1.625 \mathrm{~mm}$ (expresado por el signo negativo de los coeficientes de las cohortes 1800-49 y 1850-99) con respecto a las cohortes de referencia, controladas por la edad y el municipio de residencia (es decir, si estas variables permanecieran constantes). Recordemos, no obstante, que la interpretación de esos coeficientes no es sencilla puesto que se refieren al impacto sobre el logaritmo de un cociente de probabilidades $(Y)$. La transformación exponencial de esos resultados, en la columna $\operatorname{Exp}(B)$ sí es directamente interpretable como la diferencia en la probabilidad de marcar $1.625 \mathrm{~mm}$ con respecto a las cohortes de referencia. De esta forma, 0.790 para las cohortes 1850-99 significa un descenso del $21 \%$ en la probabilidad de llegar a la marca con respecto a las cohortes nacidas antes de $1800^{46}$. La agrupación de cohortes 1800-1849 también presenta un riesgo menor de marca pero en este caso el valor no es estadísticamente significativo. En contraste, la agrupación posterior a $1900(\operatorname{Exp} B=1.340)$ aumenta un 34\% la probabilidad de marcar $1.625 \mathrm{~mm}$.

CUADRO 7

ANÁLISIS LOGÍSTICO DE TENDENCIAS DE ESTATURA A LARGO PLAZO

\begin{tabular}{|c|c|c|c|c|c|c|}
\hline & B & S.E. & Wald & df & Sig. & $\operatorname{Exp}(B)$ \\
\hline Cohortes & & & 161,753 & 3 & $0,000^{*}$ & \\
\hline Anteriores a 1800 & Ref & & & & & \\
\hline $1800-49$ & $-0,116$ & 0,082 & 2,005 & 1 & 0,157 & 0,890 \\
\hline $1850-1899$ & $-0,236$ & 0,067 & 12,536 & 1 & $0,000^{*}$ & 0,790 \\
\hline Posteriores a 1900 & 0,293 & 0,065 & 20,099 & 1 & $0,000^{*}$ & 1,340 \\
\hline \multicolumn{2}{|l|}{ Edad } & & 64,460 & 2 & $0,000^{*}$ & \\
\hline 19 & Ref & & & & & \\
\hline 20 & 0,309 & 0,049 & 39,279 & 1 & $0,000^{*}$ & 1,362 \\
\hline $21+$ & 0,395 & 0,050 & 61,231 & 1 & $0,000^{*}$ & 1,484 \\
\hline \multicolumn{7}{|l|}{ Municipio } \\
\hline Montefrio & Ref & & & & & \\
\hline Santa Fe & 0,007 & 0,037 & 0,040 & 1 & 0,842 & 1,007 \\
\hline Constant & 0,102 & 0,071 & 2,047 & 1 & 0,152 & 1,108 \\
\hline
\end{tabular}

Nota: Las cohortes nacidas antes de 1800 y una marca de $1.625 \mathrm{~mm}$ sirve de referencia.

* Coeficientes significativos con un nivel de confianza del $95 \%$.

46 Si un grupo de cohortes determinado tuviese la misma probabilidad de alcanzar el registro que la agrupación de referencia, el valor obtenido sería 1. 
Los valores de la variable Edad son los esperados: a mayor edad, tomando la de 19 años como referencia, aumenta el riesgo de marcar $1.625 \mathrm{~mm}$ puesto que, como se ha teorizado ampliamente, el proceso de crecimiento en el pasado se prolongaba más allá de lo que ahora se considera normal en las sociedades desarrolladas (en torno a los 18 años para los varones $^{47}$ ). Respecto a la variable Municipio, no se aprecian diferencias significativas entre Montefrío y Santa Fe.

Encontramos, por tanto, que controladas variables potencialmente influyentes en la estatura como la edad y el municipio de residencia (léase en términos socioeconómicos y epidemiológicos) y asumiendo que no se produjeron cambios sustanciales en la distribución de estaturas (esto es procesos importantes de divergencia entre los segmentos de la distribución) se habría producido un ciclo de descenso de la estatura durante el siglo XIX que resulta estadísticamente significativo para la segunda mitad de esa centuria.

Estas primeras evidencias, reconociendo su parcialidad, se muestran coherentes con otras que de manera indirecta también proceden de las fuentes militares. Así, al reconstruir la evolución del mínimo exigido para poder realizar el servicio militar obligatorio desde 1741 (Cuadro 4) hemos observado un descenso progresivo que llevó ese listón desde el 1,62 m a mediados del siglo XVIII al 1,54 m a comienzos del siglo XX (8 cm menos). La cuestión es que a partir de esa aproximación no podíamos sacar conclusiones definitivas sobre el nivel de vida biológico puesto que las variaciones en el mínimo exigido siempre podrían responder a una necesidad creciente de tropas. En este sentido, los nuevos resultados obtenidos parecen confirmar que, aparte de que disminuyesen los requerimientos físicos ante determinadas coyunturas en las que se necesitaron más soldados, esa disminución también podría responder a un descenso progresivo de la estatura media entre mediados del siglo XVIII y comienzos del siglo XX.

Sobre si ese descenso estructural afectó al conjunto de la población o a determinados sectores, es algo que de momento sólo puede ser respondido a partir de las series continuas de estatura que arrancan en el siglo XIX. A este respecto, en primer lugar, hay que destacar que las medias de los segmentos de la distribución de estaturas (correspondientes cada uno al 25\% de la distribución; Gráfico 3) siguen una evolución bastante paralela a lo largo del periodo analizado. En segundo lugar, la observación conjunta de los Gráficos 2 y 3 indica que los ciclos cortos de descenso dentro del periodo de crisis estructural de la segunda mitad del siglo XIX afectaron de manera especial tanto al segmento superior de estaturas como al inferior. El 50\% restante de la población observada, agrupada en los segmentos intermedios (Q2 y Q3) experimentó un estancamiento de su estatura media para las generaciones nacidas entre 1857 y 1895 aproximadamente. No obstante, por último, cabe destacar que el periodo más crítico para el estado nutricional en las dos comunidades estudiadas (1860-1890) (Gráfico 2) coincide con las diferencias más notables entre el segmento inferior de la distribución y el resto (al respecto, obsérvese como la estatura media del 25\% más bajo de la distribución, Q1, no superó el 1,55 m hasta principios del siglo xx).

En el Cuadro 8, donde ponemos apellidos y condición social a los anteriores datos para la localidad de Montefrío, comprobamos que efectivamente los ciclos de descenso de la estatura afectaron a todos los sectores de la comunidad si bien las diferencias se mantuvieron en niveles muy altos hasta finales del siglo XIX y, tras un proceso de convergencia durante las primeras décadas del XX, volvieron a aumentar para las generaciones nacidas entre 1930 y 1944. Cabe destacar, por último, dos aspectos. El primero, que los hijos de las clases acomo dadas siempre estuvieron en estándares de estatura moderna. Y el segundo, que estos pudie-

47 Tanner (1986); Bogin (1988). 


\section{GRÁFICO 2}

ESTATURA MEDIA MASCULINA (MM. Y COHORTE DE NACIMIENTO) MEDIAS MÓVILES QUINQUENALES MONTEFRÍO Y SANTA FE (1857-1944)

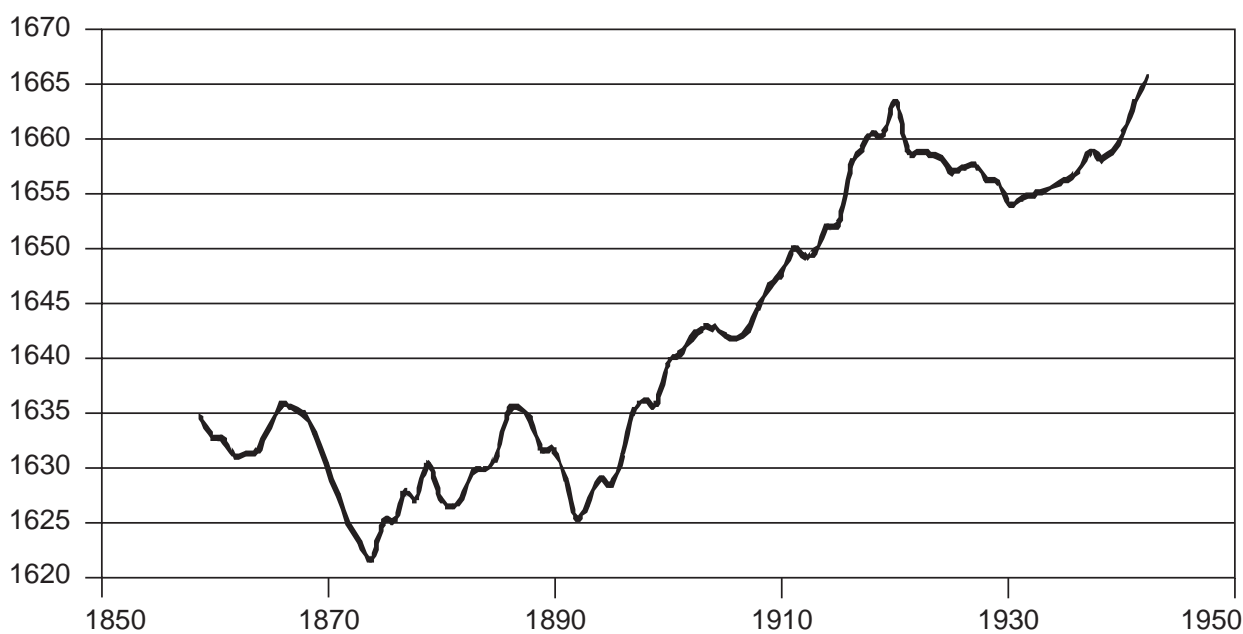

GRÁFICO 3

EVOLUCIÓN DE LA ESTATURA MEDIA POR SEGMENTOS DE POBLACIÓN (25\%) MONTEFRÍO Y SANTA

FE (1857-1944) (MM. Y COHORTE DE NACIMIENTO). MEDIAS MÓVILES QUINQUENALES

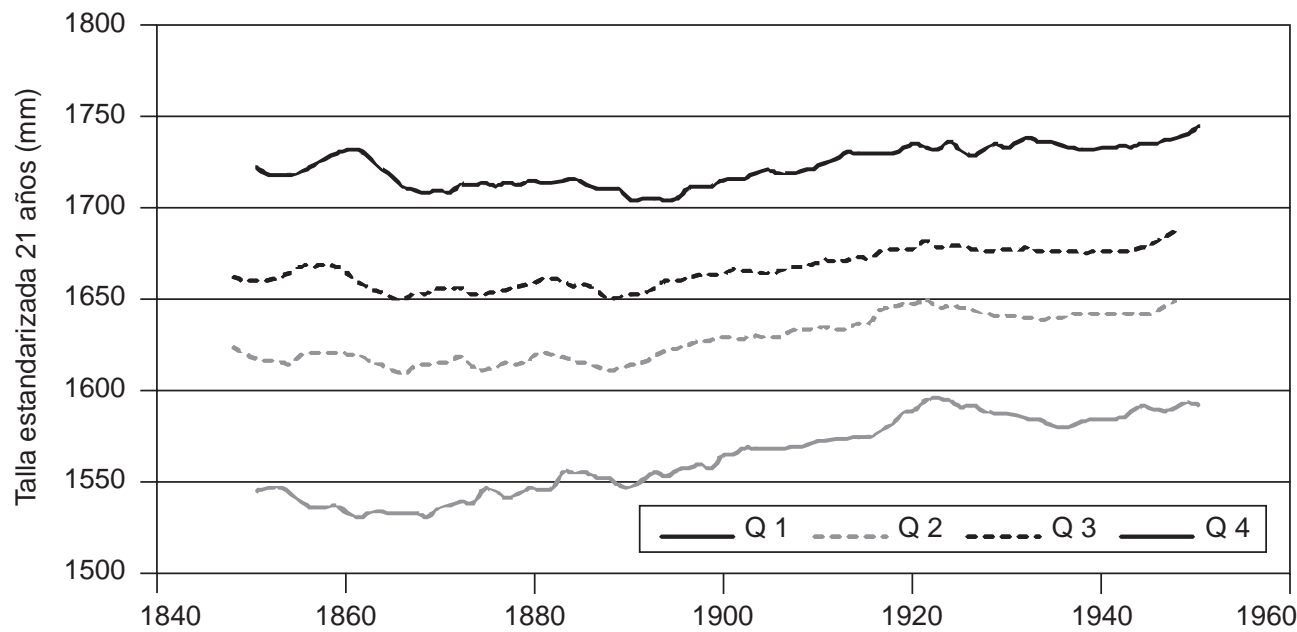

ron ser particularmente sensibles a situaciones puntuales de presión ambiental o cambios en el contexto epidemiológico (menos probable serían las situaciones de escasez entre estas clases acomodadas rurales) como así lo atestigua el descenso de estatura que todos ellos experimentaron en Montefrío entre 1830 y 1900. Ambos aspectos constituyen líneas de investigación realmente interesantes en España a corto plazo. 
CUADRO 8

ESTATURA MEDIA MASCULINA COMPARADA (COMUNIDAD Y FAMILIAS ACOMODADAS) (MM.) MONTEFRÍO, 1830-1944

\begin{tabular}{lccccc}
\hline \multirow{2}{*}{ Adscripción familiar } & \multicolumn{5}{c}{ Cohortes de nacimiento (número de observaciones) } \\
\cline { 2 - 6 } & $\mathbf{1 8 3 0 - 5 9}$ & $\mathbf{1 8 6 0 - 8 9}$ & $\mathbf{1 8 9 0 - 1 9 0 9}$ & $\mathbf{1 9 1 0 - 2 9}$ & $\mathbf{1 9 3 0 - 4 4}$ \\
\hline García-Valdecasas & $1714(7)$ & $1699(16)$ & $1689(8)$ & & $1691(3)$ \\
Ruiz-Fuensalida & $1757(2)$ & $1685(4)$ & $1649(2)$ & & \\
Alba & $1694(7)$ & $1691(7)$ & $1666(7)$ & $1701(3)$ & $1708(3)$ \\
Rico & $1696(11)$ & $1691(7)$ & $1657(10)$ & $1642(8)$ & $1676(9)$ \\
\hline Media familias acomodadas & $1715(27)$ & $1691(34)$ & $1665(27)$ & $1671(11)$ & $1692(12)$ \\
\hline Media comunitaria & 1648 & 1632 & 1636 & 1654 & 1658 \\
\hline
\end{tabular}

Queremos destacar también la consistencia general de estos resultados con los estudios de mortalidad que sobre las mismas comunidades se han realizado y que muestran una relación inversa de este indicador (aun expresado en tasas brutas) con la estatura ${ }^{48}$. Además, el probable deterioro del estado nutricional neto de la población nacida durante el siglo XIX (particularmente el grupo de cohortes 1850-1900) se enmarca en el escenario general de mortalidad dibujado para el conjunto del país en dicho periodo. Ese escenario, bien ilustrado por indicadores como la mortalidad infantil y la esperanza de vida, corresponde más a uno del Antiguo Régimen que a uno de la sociedad moderna. Durante la segunda mitad del siglo XIX las tasas brutas de mortalidad se mantuvieron invariablemente altas en torno al 30 por mil ${ }^{49}$ y los cocientes de mortalidad de menores de diez años superaron sistemáticamente el 400 por mil hasta comienzos del siglo $x x^{50}$. A resultas de ello, la esperanza de vida no experimentó ningún progreso sensible e incluso en periodos concretos como 1863-1882 podría haber sufrido un ligero descenso (desde los 29,7 a los 29,1 años) ${ }^{51}$.

Las implicaciones para el debate general en historia económica nos parecen interesantes porque si damos por buenos los trabajos que confirman un proceso de crecimiento económico durante ese periodo ${ }^{52}$, merecería la pena indagar en los factores estructurales que hicieron que ese crecimiento no redundara en beneficio del nivel de vida biológico, más allá de la amplia batería de causas propuestas en diferentes trabajos, a saber, deterioro de la dieta y aumento de los niveles de autoexplotación campesina, posible aumento de los niveles de endeudamiento asociado a la capitalización de las explotaciones en el marco de mercantilización de las economías campesinas, desmantelamiento del sistema de bienes y usos comunales, y pérdida de poder adquisitivo de las clases bajas por el descenso de los salarios reales ${ }^{53}$ a lo que en nuestra opinión habría que sumar el proceso de crecimiento demográfico sostenido que se estaba viviendo desde el siglo XVIII.

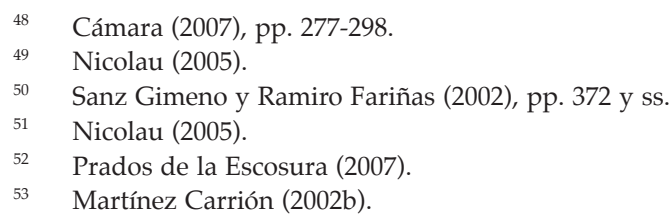




\section{Conclusiones}

El análisis logístico de tendencia de la estatura generacional en agrupaciones amplias de cohortes resulta una aproximación atractiva para el estudio de la evolución de los niveles de vida biológicos a largo plazo. Esta aplicación metodológica permite incluir en el análisis datos antropométricos que son de difícil integración en series temporales por el menor detalle y calidad del registro durante el Antiguo Régimen. Además abre la posibilidad de referenciar las series antropométricas elaboradas desde 1840 al ampliarse nuestra perspectiva hacia el pasado. En concreto podemos disponer de información sobre las cohortes masculinas que fueron quintadas durante el reinado de Carlos III, nacidas por tanto desde mediados del siglo XVIII e incluso antes.

Se debe reconocer que los resultados obtenidos constituyen de momento una evidencia parcial al provenir de dos localidades y de una metodología cuyas limitaciones y riesgos ya han sido expuestos. En este sentido, la naturaleza de los datos anteriores a los alistamientos de mediados del siglo XIX, salvo excepciones, siempre conllevará un grado variable de incertidumbre en cuanto a la fiabilidad de las estimaciones realizadas sobre ellos. Esa incertidumbre, básicamente, está generada por el desconocimiento de la evolución concreta de los segmentos de la distribución de estatura que, por otra parte, presenta gran estabilidad y paralelismo entre mediados del siglo XIX y mediados del XX. Apoyándonos en esta evidencia, nuestra impresión es que, incluso admitiendo una eventual divergencia en la evolución de la estatura media de distintos segmentos de la población, habría que admitir que al menos un sector importante de la misma experimentó un deterioro sensible de su estado nutricional neto durante el siglo XIX con respecto al siglo XVIII (argumento que por primera vez no se basa en analogías con procesos ocurridos en otros países).

De la existencia de ciclos de estatura en el medio rural durante los siglos XIX y xx ya sabíamos, descartando la idea de inmovilismo del medio rural también en términos de niveles de vida. Pero, además, referenciados con respecto a las cohortes nacidas durante la segunda mitad del siglo XVIII, nuestros resultados evidencian un deterioro del estado nutricional de la población coincidiendo con la consolidación del estado liberal y con una fase expansiva del capitalismo agrario decimonónico entre 1850 y 1900. En el caso de las dos comunidades analizadas, ese ciclo coincide con un proceso de especialización agrícola, crecimiento agrario y crecimiento demográfico notables.

El valor añadido de estas evidencias locales consiste en que, referenciadas a nivel nacional, sus registros de estatura se encuentran en un nivel medio durante el siglo XIX. Por tanto, también los resultados para periodos anteriores resultarían representativos o hasta cierto punto indicativos de lo que podría haber ocurrido en el conjunto del medio rural español aún a sabiendas de la heterogeneidad del mismo (heterogeneidad que en cualquier caso pudo derivar en respuestas y ciclos diferenciados a corto e incluso a medio plazo pero no, creemos, a largo plazo ni procediendo por agrupaciones de cohortes tan amplias).

En el fondo, tampoco debería sorprender demasiado el sentido de las tendencias trazadas en este trabajo ya que resultaba difícil de aceptar que la población masculina española hubiera sido secularmente tan baja como mostraban los datos antropométricos de las décadas centrales del siglo XIX. Bien es cierto que hasta el momento no podíamos ir más allá de las conjeturas puesto que no disponíamos de información antropométrica para las generaciones nacidas con anterioridad a 1840 y por tanto no podía asegurarse que los registros del Ochocientos informaran sobre una crisis estructural del estado nutricional de la población. En consecuencia, de confirmarse estas tendencias para otros ámbitos, habrá que reconocer que la estatura en España en las postrimerías del Antiguo Régimen se habría aproximado más a 
estándares modernos que la registrada durante las décadas centrales del siglo XIX. En ese caso se abriría una interesante línea de investigación que tendría que plantear que, como en otros aspectos, se pudo producir un proceso de divergencia antropométrica con otras regiones del continente durante la transición a la sociedad moderna incluso reconociendo que los indicadores biológicos de partida fueran bastante pobres.

Por último, puesto que no se ha ensayado hasta ahora un modelo explicativo "estructural" para España cuando precisamente las condiciones ambientales y la realidad física de la mayoría de sistemas agrarios mediterráneos se prestan especialmente a ello, creemos que nuestra propuesta metodológica puede animar a la obtención de nuevos resultados y a la elaboración progresiva de aquel por parte de la Historia Económica y la Demografía Histórica española.

\section{Bibliografía}

ABELLA, Fermín (1915): Manual de Quintas, Madrid, Imprenta de El Consultor.

A'HEARN, Brian (2004): “A Restricted Maximum Likelihood Estimator for Historical Height Samples", Economics and Human Biology, 2, pp. 5-19.

BARCIELA, Carlos; GIRÁLDEZ, Jesús; GEHR y LÓPEZ, Inmaculada (2005): "Sector agrario y pesca", en CARRERAS, Albert y TAFUNELL, Xavier (coords.), Estadísticas Históricas de España, Segunda ed., Fundación BBVA, pp. 246-356.

BENGTSSON, Tommy y SAITO, Osamu (2000) (eds.): Population and Economy. From hunger to modern economic growth, Oxford, Oxford University Press.

BOGIN, Barry (1988): Patterns of Human Growth, Cambridge University Press.

BRINGAS GUTIÉRREZ, Miguel Ángel (2000): La productividad de los factores en la agricultura española (1752-1935), Servicio de Estudios del Banco de España, Estudios de Historia Económica, 39.

CÁMARA, Antonio David (2006): “Fuentes antropométricas en España: problemas metodológicos para los siglos XVIII y XIX", Historia Agraria, 38, pp. 575-582.

-(2007): Niveles de vida en el medio rural de Andalucía Oriental (1750-1950), Tesis doctoral, Universidad de Granada.

—(2007b): “La paradoja de la modernización en España: el descenso de la estatura en el medio rural durante la fase expansiva del primer capitalismo agrario", III Congreso Internacional de la Asociación Mexicana de Historia Económica, Cuernavaca, México.

—(2009): “Long-Term trends in Height in Rural Eastern Andalussia (1750-1950), Historia Agraria, 47 , pp. 45-67.

CHALLÚ, Amilcar (2009): “Agricultural Crisis and Biological Well-Being in Mexico, 17301835", Historia Agraria, 47, pp. 21-44.

COLE, Tim J. (2000): "Galton's midparent height revisited", Annals of Human Biology, 27, pp. 401-405.

-(2003): "The secular trend in human physical growth: a biological view", Economics and Human Biology, 1, pp. 161-168.

CRAFTS, Nicolas F.R. (1997): “Some Dimensions on Quality of Life during the British Industrial Revolution", Economic History Review, 50(4), pp. 617-639.

CUFF, Timothy (2005): The hidden cost of economic development. The biological standard of living in antebellum Pennsylvania, Burlington, Ashgate.

FEIJÓO, Albino (1996): Quintas y protesta social en el siglo XIX, Madrid, Ministerio de Defensa. 
FLOUD, Roderick; WATCHER, Kenneth y GREGORY, Annabel (1990): Height, health and history: nutritional status in the United Kingdom 1750-1980, Cambridge, Cambridge University Press.

GARCÍA MONTERO, Héctor (2009): “Antropometría y niveles de vida en el Madrid rural, 1837-1915", Historia Agraria, 47, pp. 95-117.

GÓMEZ MENDOZA, Antonio y PÉREZ MOREDA, Vicente (1985): “Estatura y nivel de vida en la España del primer tercio del siglo XX", Moneda y Crédito, 174, pp. 29-64.

GÓMEZ OLIVER, Miguel y GONZÁLEZ DE MOLINA, Manuel (1983): Transferencia de propiedad y desamortización: el caso de Santa Fe (1760-1894), Santa Fe, Ayuntamiento de Santa Fe.

GÓMEZ RUIZ, Manuel y ALONSO JUANOLA, Vicente (1991): El Ejército de los Borbones, t. II, Madrid, Servicio Histórico Militar y Ministerio de Defensa.

GONZÁLEZ DE MOLINA, Manuel y GUZMÁN CASADO, Gloria (2006): Tras los pasos de la insustentabilidad. Agricultura y medio ambiente en perspectiva histórica (s. XVII-XX), Barcelona, Icaria.

JIMÉNEZ GUERRERO, José (2001): El reclutamiento militar en el siglo XIX. Las quintas en Málaga (1837-1868), Málaga, Universidad de Málaga.

KOMLOS, John (1994) (ed.): Stature, living standards, and economic development. Essays in Anthropometric History, Chicago and London, University of Chicago Press.

-(2004): "How to (and Not to) Analyze Deficient Height Samples", Historical Methods, 37, 1, pp. 160-173.

KOMLOS, John y KIM, Jae H. (1990): “Estimating trends in historical heights", Historical Methods, 23, pp. 116-120.

LANA BERASAIN, José Miguel (2002): “Jornales, salarios, ingresos. Aproximación a la evolución de los niveles de vida desde la Navarra rural, 1801-1935", en MARTÍNEZ CARRIÓN, José Miguel (ed.), El nivel de vida en la España rural, siglos XVIII-XX. Alicante, Universidad de Alicante, pp. 183-234.

MARTÍNEZ CARRIÓN, José Miguel (2001): Estatura, salud y bienestar en las primeras etapas del crecimiento económico español. Una perspectiva comparada de los niveles de vida, Documentos de trabajo de la AEHE, Núm. 0102.

-(2002) (ed.): El nivel de vida en la España rural, siglos XVIII-XX, Alicante, Universidad de Alicante.

- (2002b): "El nivel de vida en la España rural, siglos XVIII-XX. Nuevos enfoques, nuevos resultados", en MARTÍNEZ CARRIÓN, José Miguel (ed.), El nivel de vida en la España rural, siglos XVIII-XX, Alicante, Universidad de Alicante, pp. 16-72.

MARTÍNEZ CARRIÓN, José Miguel y MORENO LÁZARO, Javier (2007): “Was there an urban height penalty in Spain, 1840-1913?", Economics and Human Biology, 5, pp. 144-164.

MARTÍNEZ CARRIÓN, José Miguel y PÉREZ CASTEJÓN, Juan José (2000b): “On the height of Spanish recruits during the early phases of modern economic growth", Jahrbuch für Wirtschaftsgeschichte, 1, pp. 95-112.

-(2002): "Creciendo con desigualdad. Niveles de vida biológicos en la España rural mediterránea desde 1840", en MARTÍNEZ CARRIÓN, José Miguel (ed.), El nivel de vida en la España rural, siglos XVIII-XX, Alicante, Universidad de Alicante, pp. 405-460.

MARTÍNEZ CARRIÓN, José Miguel y PUCHE GIL, Javier (2009): “Alfabetización, bienestar biológico y desigualdad: la Comunidad Valenciana, 1850-1970", Historia Agraria, 47, pp. 167-186.

MARTÍNEZ LÓPEZ, David (1996): Tierra, herencia y matrimonio, Jaén, Universidad de Jaén.

MARTÍNEZ MARTÍN, Manuel (1995): Revolución Liberal y Cambio Agrario en la Alta Andalucía, Granada, Universidad de Granada. 
MORENO LÁZARO, Javier (2002): “¿Fomentó el capitalismo agrario la desigualdad? Salarios y niveles de vida en Castilla la Vieja 1751-1861", en MARTÍNEZ CARRIÓN, José Miguel (ed.), El nivel de vida en la España rural, siglos XVIII-XX. Alicante, Universidad de Alicante pp. 75-112.

-(2006): "El nivel de vida en la España atrasada entre 1800 y 1936. El caso de Palencia", Investigaciones de Historia Económica, 4, pp. 9-50.

NICOLAU, Roser (2005): "Población, salud y actividad", en CARRERAS, Albert y TAFUNELL, Xavier (coords.), Estadísticas Históricas de España. Siglos XIX y XX, Segunda ed., Fundación BBVA, pp. 79-154.

PORTUGUÉS, José Antonio (1764): Recopilación de Ordenanzas Militares, Madrid.

PRADOS DE LA ESCOSURA, Leandro (2007): “Growth and structural change in Spain, 18502000: a european perspective", Revista de Historia Económica - Journal of Iberian and Latin American Economic History, 1, pp. 147-177.

PUCHE GIL, Javier (2005): “Niveles de vida biológicos en el País Valenciano. 1840-1960. Evidencias antropométricas", VIII Congreso de la AEHE, Santiago de Compostela.

PUJOL, Josep; GONZÁLEZ DE MOLINA, Manuel; FERNÁNDEZ, Lourenzo; GALLEGO, Domingo y GARRABOU, Ramón (2001) (eds.): El pozo de todos los males. Sobre el atraso en la agricultura española contemporánea, Barcelona, Crítica.

QUARANTA, Luciana (2007): “A case study of missing historical heights in Friuli (Italy). Estimation using multiple imputation“, Working Paper N.1, Dipartimento di Scienze Statistiche dell'Universita' degli Studi di Udine.

QUIROGA, Gloria (2002): “Estatura y condiciones de vida en el mundo rural español, 18931954", en MARTÍNEZ CARRIÓN, José Miguel (ed.), El nivel de vida en la España rural, siglos XVIII-XX, Alicante, Universidad de Alicante, pp. 461-494.

RAMÓN i MUNÕZ, Josep María (2009): “Bienestar biológico y crecimiento agrario en la Cataluña rural, 1840-1936", Historia Agraria, 47, pp. 119-142.

RUBIN, Donald B. (1987): Multiple imputation for non-response in surveys, New York, John Wiley \& Sons.

SANZ GIMENO, Alberto y RAMIRO FARIÑAS, Diego (2002): “Infancia, mortalidad y niveles de vida en la España interior. Siglos XIX y XX", en MARTÍNEZ CARRIÓN, José Miguel (ed.), El nivel de vida en la España rural, siglos XVIII-XX, Alicante, Universidad de Alicante, pp. 359-403.

SIMPSON, James (1995): Spanish agriculture. The long Siesta, 1765-1965, Cambridge, Cambridge University Press.

SPIJKER, Jeroen; PÉREZ DÍAZ, Julio y CÁMARA, Antonio David (2008): “Cambios generacionales de estatura en la España del siglo XX a través de la Encuesta Nacional de Salud", Revista de Estadística Española, 169, pp. 571-604.

TANNER, James M. (1986): “Growth as a target-seeking-function. Catch up and catch-down growth in man", en FALKNER, Frank and TANNER, James M. (eds.), Human Growth. A comprehensive treatise, New York, Plenum Press, 3 Vols., pp. 167-180.

TORTELLA, Gabriel (1992): “La historia económica de España en el siglo XIX: un ensayo comparativo con los casos de Italia y Portugal", en PRADOS DE LA ESCOSURA, Leandro y ZAMAGNI, Vera (eds.), El desarrollo económico en la Europa del Sur: España e Italia en perspectiva histórica, Madrid, Alianza, pp. 56-80.

WATCHER, Kenneth W. and TRUSELL, James (1982): "Estimating historical heights", Journal of American Statistical Association, 77, pp. 279-293. 